\title{
A Study of Scheduling Algorithms in WiMAX
}

\author{
Surinder Singh, Gaurav Kumar \\ Chandigarh Engineering College Landran (Mohali) Punjab,India \\ Department of Electronics and communication Engineering ,Chandigarh Engineering \\ College Landran (Mohali) Punjab, India
}

\begin{abstract}
As we know wireless broadband internet is very popular in modern era. For wireless Broadband internet, speed is big issue and it is a hot research area for researchers to focus on this area. WiMAX (worldwide interoperability for Microwave Access) networks are the networks which are capable for providing BWA(broadband wireless Access) with high speed. A WiMAX has one base station (BS) and one or more subscriber station (SS). IEEE 802.16 standard gives QOS parameter to traffic. It offers five categories to prioritize the traffic. Five categories are the classes in which traffic can be divided. These five classes are VoIP, MPEG video, FTP, MPEG video and web browsing. Once the packets are classified according the IEEE802.16 standards, these packets are placed into multipriority queue. In this queue these packets are placed from high priority to low priority. After that the application of scheduler comes into the picture. A Scheduler executes the process according to priority of processes. Scheduling plays an important role in WiMAX. For Scheduling there are algorithms which manage the incoming packets for each process of various nodes. These Algorithms are responsible for processing and executing the process in ready Queue. Scheduling algorithms deals with many parameters like turnaround time, waiting time and execution time etc. This paper deals with features of scheduling algorithms and also classification of
\end{abstract}

scheduling algorithms. This paper also suggests a new scheduling algorithm.

\section{Introduction}

WiMAX (worldwide interoperability for Microwave Access) networks are the networks which are responsible for providing many services like video, data and voice. The WiMAX technology satisfies the modern need of broadband internet through wireless access. For managing all these services through WiMAX, IEEE802.16 gives QOS (Quality of Service) parameter [1]. This parameter gives five classes to manage the traffic of VoIP, MPEG video, FTP, MPEG video and web browsing. In WiMAX, a fundamental challenge is to achieve high QOS so that various parameters like waiting time, end to end delay can be minimized and other parameter like execution time, network utilization and throughput can be increased.

\section{WiMAX Architecture}

The IEEE 802.16 gives a standard to the WiMAX. It consists of one BS (base station) and one or more SSs (Subscriber stations). The other language of Base station is the backbone of the WiMAX. It is similar to the cell phone tower [2]. The range of the base station is the radius of 6 miles. The subscriber station consists of one or more users.

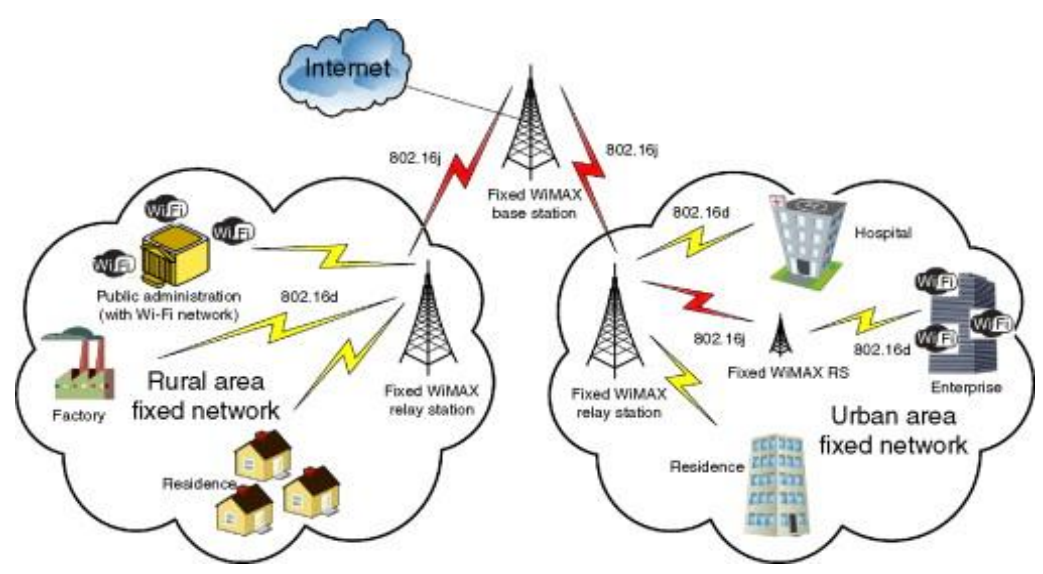

Figure 1. WiMAX 


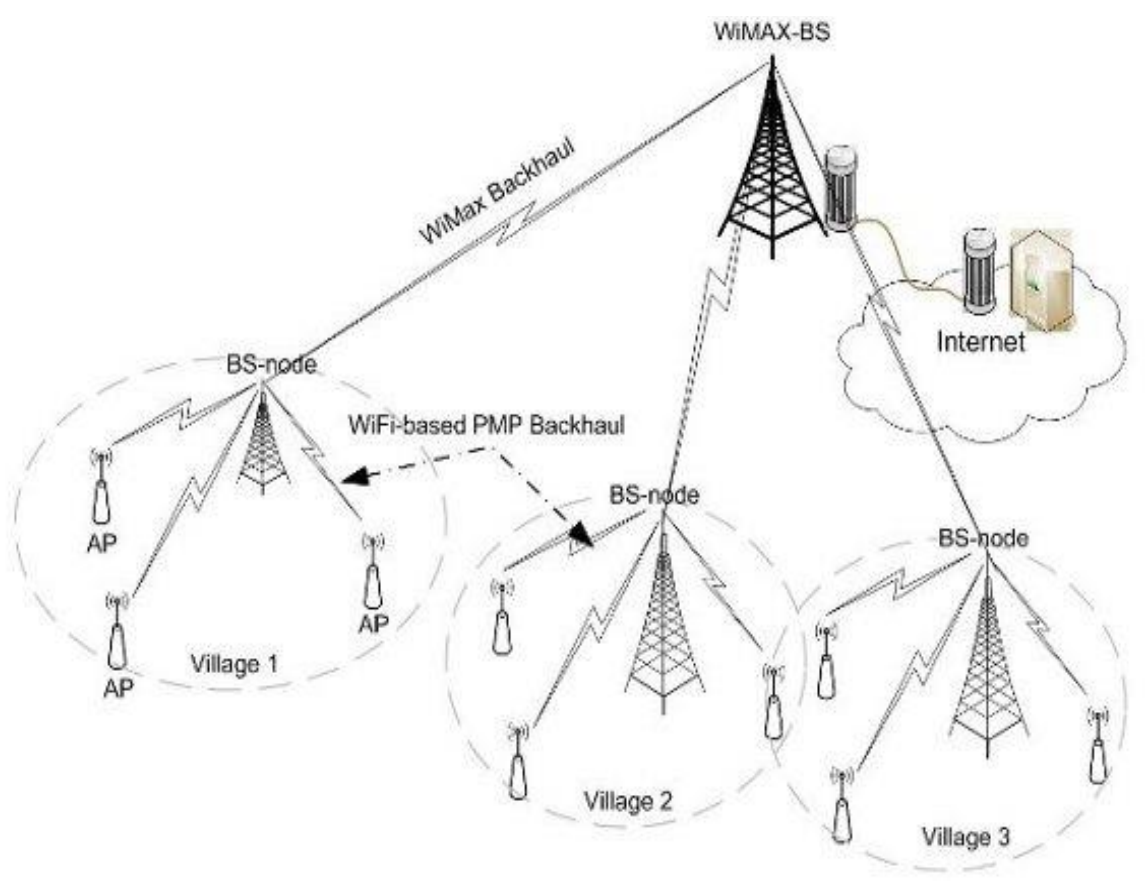

Figure 2. WiMAX Architecture

The Figure 2 shows WiMAX Architecture The base node of all three tower are attached to base station through WiMAX link.

\section{Algorithms Used For Scheduling}

Scheduling algorithms are basically of two types. In first type the execution of process cannot stop until its execution are called Non Preemptive scheduling algorithms for example FCFS (First come first serve), SJF (shortest Job First), SP (Strict Priority) [3]. In second type of process execution of process can be stopped before their executions are called Preemptive scheduling algorithms for example RR (Round Robin) etc.

\subsection{FCFS (First come First Serve) Scheduling algorithm}

It is a Non Preemptive type Scheduling Algorithm. It uses FIFO Strategy. The process first enter in the ready queue will execute first. Any blocked process can be put into the block pool. As all the processes are unblock so they are put into the tail of ready queue. The advantage of FCFS [4] is that as no method of prioritization is followed, hence there is no problem of starvation. The disadvantage of FCFS is that it shows poor performance (see Figure 3, the Gantt chart for FCFS scheduling).

\begin{tabular}{cc} 
Process & Burst Time \\
\cline { 1 - 1 }$P_{1}$ & 53 \\
$P_{2}$ & 17 \\
$P_{3}$ & 68 \\
$P_{4}$ & 24 \\
chart is: &
\end{tabular}

The Gantt chart is:

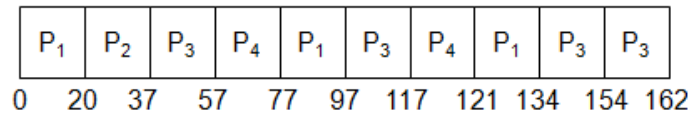

Figure 3. FCFS Gantt Chart

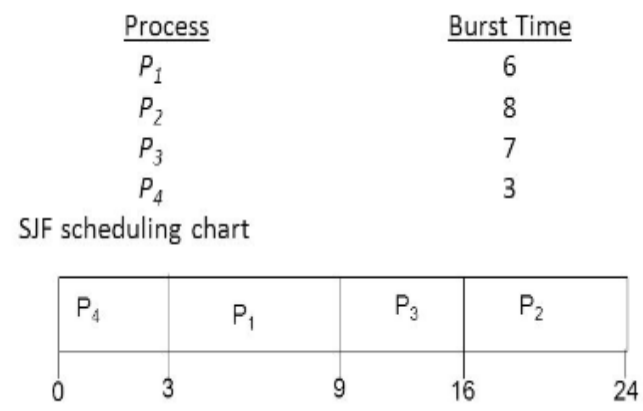

Figure 4. Processes without SJF 


\subsection{SJF (Shortest Job First) algorithm}

As the name indicates that in this type of scheduling algorithm the shortest job will execute first. The above Figure 4 shows the processes without SJF, that is the job with random burst time. Above is the process with SJF, that is job with less burst time will execute first and so on [5].

\subsection{Strict Priority Scheduling or Priority Scheduling}

Non-preemptive type scheduling that is as soon as the higher priority jobs are executed the lower jobs cannot execute In this scheduling the execution of jobs are done on the basis of priority assigned to the jobs. The jobs assigned with highest priority will execute first and jobs with low priority will execute
After higher priority job [6]. The Figure 5 shows Strict priority Scheduling. The incoming packets are classified into two categories. The higher priority jobs are placed into the higher priority queue and lower priority queue placed into the lower priority queue. The higher priority jobs are executing first and lower priority jobs are executed after higher priority jobs.

\subsection{Round Robin Scheduling}

It works in rounds. It is preemptive type scheduling. It serves the first packet in each priority queue in sequence according to their precedence till all the queues are served. After completion of first packet in each queue it restarts over to the second packet in each queue (see Figure 6 Round Robin Scheduling).

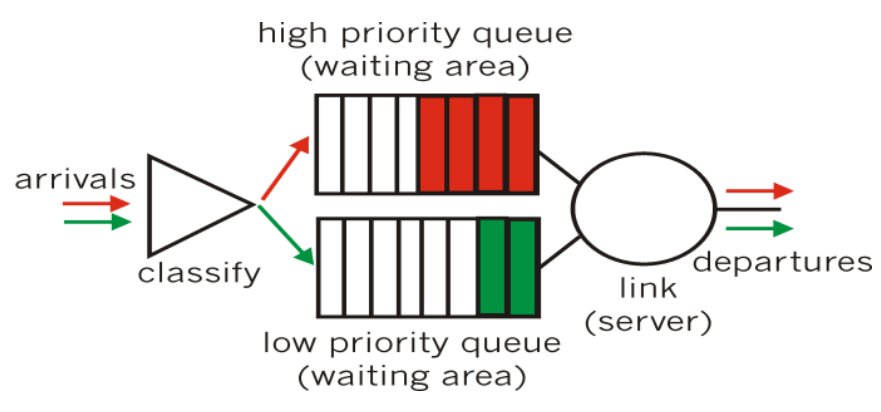

Figure 5. Strict priority Scheduling

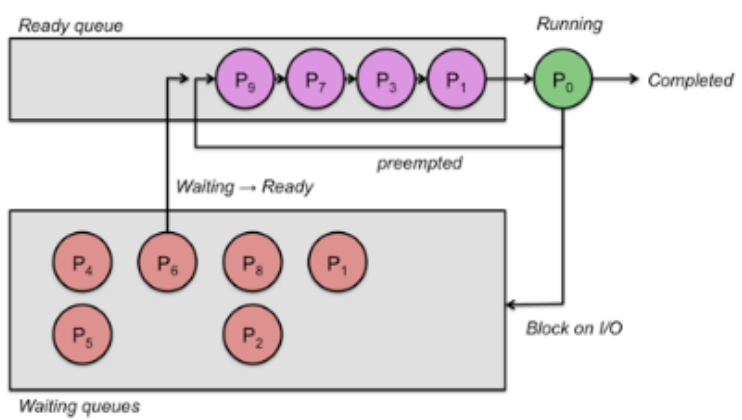

Figure 6. Round Robin Scheduling

\subsection{Greedy -Latency Scheduling}

Greedy algorithm is a distributed, Like the random algorithm, It executes on individual node. Each node first recursively tasks all nodes in its local sub tree [7]. Once the sub tree has been recursively tasked, the node then tasks itself and estimates the total latency within its sub tree (but not for the entire network). This is done by simulating the sub tree with the given tasking schedule. Afterwards, the node de-tasks itself and re-estimates the total latency with the new schedule [6], [7], [9]. The node finally chooses the strategy that yields the lower latency. Figure 7 shows the greedy Latency Scheduling. 


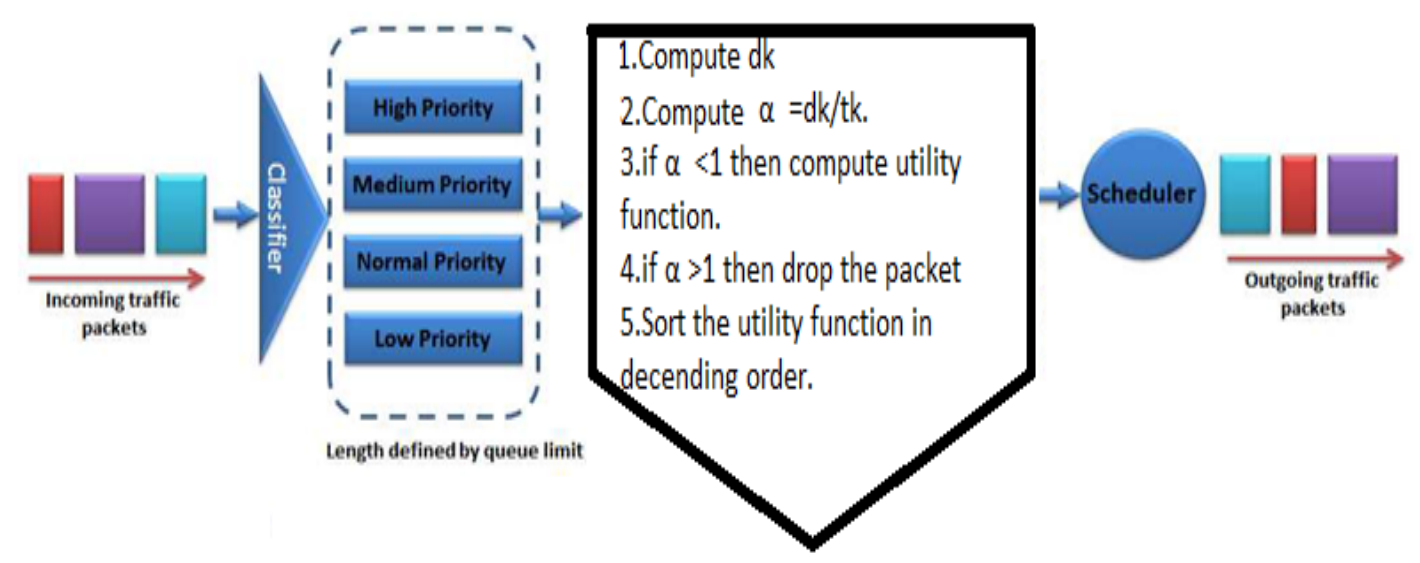

Figure 7. Greedy Latency Scheduling

\section{Proposed Algorithm}

In this paper we propose a new algorithm which is the addition in the greedy Latency scheduling. We propose an algorithm which first calculates distance of nodes can be calculated using distance formula. After that the burst time of each user can be calculated [8][9]. After that the greedy latency algorithm can be used. In this algorithm the utility values using greedy latency algorithm using this formula utility function for kth user is given by:

$$
\begin{aligned}
& \mathrm{U}(\mathrm{d}, \mathrm{t}, \gamma)= \\
& \left\{\begin{array}{cc}
\arg \operatorname{maxk}(\mathrm{dk} / \mathrm{Tk} & \left.\gamma^{-} \mathrm{k}\right) \quad \text { for } \mathrm{dk} / \mathrm{Tk}<1 \\
\text { Packet drop } & \text { for dk/Tk }>1 \\
\text { Tk>0, dk>0 } & \forall \mathrm{k} \in\{1,2,3, \ldots, \mathrm{N}\}
\end{array}\right.
\end{aligned}
$$

\section{Conclusion}

We come to the conclusion that our proposed approach has better performance. Results from our simulations using MATLAB shows that the proposed system provides a efficient scheduling algorithm. The results show that the average turnaround time, waiting time. This will help to increase the speed of packet delivery from base station to subscriber station. This scheduling can increase the execution of those users which are closer to the base station.

\section{References}

[1] Chandur P., Karthik R. and Sivalingam K. 2012 "Performance Evaluation of Scheduling Algorithms for Mobile WiMAX Networks" IEEE 978-1-46730906-6 pp 770-775.
[2] Chen J., W. Jiao and H. Wang, 2005 "A Service Flow Management Strategy for IEEE 802.16 Broadband Wireless Access Systems In TDD mode in Communications" ICC IEEE International Conference, vol. 5, pp. 3422-3426.

[3] Dahmouni H., El Ghazi H., Bonacci D., Sansò B. and Girard A. MAY 2010 "Improving QOS of all-IP Generation of Pre-WiMax Networks Using DelayJitter Model" Journal of telecommunications, VOL. 2, pp 99-103.

[4] Deepak H., and Nayak S. 2012, "Bandwidth Recycling In WiMAX Networks" (IJCSIT) International Journal of Computer Science and Information Technologies, Vol. 3,38523855.ISSN:0975-9646, pp.3852-3855.

[5] Dosciatti R., Godoy W., and Foronda A., 2012 "An Efficient Approach of Scheduling With Call Admission Control to Fixed WiMAX networks," Latin America Transactions, IEEE (Revista IEEE America Latina), vol. 10, no. 1, pp. 1256-1264.

[6] Gidlund M. and Wang G., March 2009 "Uplink Scheduling Algorithms for QOS Support in Broadband Wireless Access Networks" JOURNAL OF COMMUNICATIONS, VOL. 4,pp 133-142.

[7] Galshetwar G., Jayakumar A. and Mittal Y. Apr 2012 "Comparative Study Of Different Scheduling Algorithms For Wimax MAC Scheduler Design" International Journal of Engineering Research and Applications (IJERA) ISSN: 2248-9622, Vol. 2, Issue 2, pp.1031-1037.

[8] Gupta S. 2013 "Comparison of Various Scheduling Algorithms In WiMAX: A Brief 
International Journal of Digital Society (IJDS), Volume 9, Issue 4, December 2018

Review" Published in International Journal of Computer Applications (IJCA), pp 34-36.

[9] Haghani E. and Ansari N. 2008 "VoIP Traffic Scheduling In WiMAX Networks", IEEE 978-14244-2324 pp 1-8. 